\title{
Triton Tumor
}

\section{4}

This term includes two entities: benign triton tumor and malignant triton tumor.

For details $\rightarrow$ see dedicated Chaps. 36 and 168 .

(C) The Editor(s) (if applicable) and The Author(s), under exclusive license to 